\title{
Survival impact of perioperative changes in prognostic nutritional index levels after esophagectomy
}

\author{
Ryoma Haneda ${ }^{1}$ Y Yoshihiro Hiramatsu ${ }^{1,2}$ (D) Sanshiro Kawata ${ }^{1} \cdot$ Junko Honke $^{2} \cdot$ Wataru Soneda $^{1} \cdot$ \\ Tomohiro Matsumoto $^{1} \cdot$ Yoshifumi Morita ${ }^{1} \cdot$ Hirotoshi Kikuchi $^{1} \cdot$ Kinji Kamiya $^{1} \cdot$ Hiroya Takeuchi $^{1}$
}

Received: 17 March 2021 / Accepted: 15 September 2021 / Published online: 21 September 2021

(c) The Author(s) 2021

\begin{abstract}
Background The correlation between perioperative changes in nutritional status during esophagectomy and prognosis remains unclear. This study aimed to evaluate the impact of changes in prognostic nutritional index levels during the perioperative period on esophageal cancer patient survivals.

Methods From January 2009 to May 2019, 158 patients with esophageal squamous cell carcinoma were enrolled. From the time-dependent ROC analysis, the cutoff values of preoperative and postoperative prognostic nutritional index levels were 46.9 and 40.9. Patients were divided into preoperative-high group (Group H) and preoperative-low group (Group L). Then, patients in Group L were divided into preoperative-low and postoperative-high group (Group L-H) and preoperative-low and postoperative-low group (Group L-L). Long-term outcomes and prognostic factors were evaluated.

Results Patients in Group L had significantly worse overall survival than those in Group H $(p=0.001)$. Patients in Group L-L had significantly worse overall survival than those in Group L-H $(p=0.023)$. However, there was no significant difference in overall survival between Groups $\mathrm{H}$ and $\mathrm{L}-\mathrm{H}(p=0.224)$. In multivariable analysis, advanced pathological stage (hazard ratio $10.947,95 \%$ confidence interval $2.590-46.268, p=0.001$ ) and Group L-L (hazard ratio 2.171 , 95\% confidence interval $1.249-3.775, p=0.006$ ) were independent predictors of poor overall survival.

Conclusions Patients in Group L-H had a good prognosis, similar to those in Group H. This result indicated that increasing the postoperative prognostic nutritional index level sufficiently using various intensive perioperative support methods could improve prognosis after esophagectomy in patients with poor preoperative nutritional status.
\end{abstract}

Keywords Esophageal cancer · Esophagectomy $\cdot$ Prognostic nutritional index

\section{Introduction}

Esophageal cancer is the sixth leading cause of death from cancer worldwide [1]. Despite the development of multimodal therapies, its high malignant potential and poor prognosis persist as serious problems. Transthoracic esophagectomy has been recognized as a standard treatment for esophageal squamous cell carcinoma (ESCC)

Yoshihiro Hiramatsu

hiramatu@hama-med.ac.jp

1 Department of Surgery, Hamamatsu University School of Medicine, Hamamatsu, Japan

2 Department of Perioperative Functioning Care and Support, Hamamatsu University School of Medicine, 1-20-1 Handayama, Higashi-ku, Hamamatsu, Shizuoka 431-3192, Japan
[2-4]. However, it is a highly invasive surgical procedure that results in a systemic inflammatory response and poses a risk of postoperative complications [5]. Postoperative complications may induce nutritional deficiencies and organ disorders $[6,7]$. Studies have revealed that nutritional support is important to reduce the incidence of postoperative complications and shorten hospital stays $[8,9]$. Thus, the baseline immune-nutritional status in cancer patients plays a significant role in survival.

Recently, nutritional or inflammatory status markers have been identified $[10,11]$. The prognostic nutritional index (PNI), calculated based on the serum albumin level and total lymphocyte counts, reflects both inflammatory and nutritional status [12]. Previous reports have revealed that preoperative-low PNI level was associated with worse survival in patients with ESCC [13, 14]. However, the relationship 
between changes in the PNI levels from the pre- to postoperative phase and survival remains unclear.

In this study, we hypothesized that the recovery of PNI level after esophagectomy may improve patient prognosis. The correlation between nutritional and immunological status during esophagectomy and prognosis was investigated using PNI in patients with ESCC.

\section{Patients and methods}

\section{Patients}

From January 2009 to May 2019, 188 patients with ESCC at the Department of Surgery, Hamamatsu University School of Medicine, were retrospectively reviewed. All patients underwent esophagogastroduodenoscopy (EGD) and computed tomography (CT) from the neck to the pelvis to determine the clinical stage. The clinical stage was diagnosed based on the Union for International Cancer Control TNM classification of malignant tumors, 8th edition [15].
Patients who met the following criteria were enrolled in this study: (1) age $>20$ years, (2) Eastern Cooperative Oncology Group performance status of $0-1$, (3) histological diagnosis of ESCC by endoscopic biopsy, (4) no double cancer, (5) radical esophagectomy, (6) survival for at least 90 days after surgery, and (7) patients who survived and were followed up for more than 2 years. Patient ineligible for study enrollment was based on the following exclusion criteria: death within 90 days after surgery $(n=2)$, double cancer $(n=10)$, salvage surgery $(n=12)$, and interruption of follow-up within 2 years $(n=6)$. Finally, 158 patients were included in the study (Fig. 1).

\section{Determination of the cutoff values for the prognostic nutritional index}

PNI was calculated as $10 \times$ serum albumin $(\mathrm{g} /$ $\mathrm{dL})+0.005 \times$ total lymphocyte counts $\left(\right.$ per $\left.\mathrm{mm}^{3}\right)$ [12] Preoperative PNI levels were measured just before the surgery. When patients were administered neoadjuvant chemotherapy, the PNI level was measured after this treatment. Postoperative PNI levels were obtained 1 month after

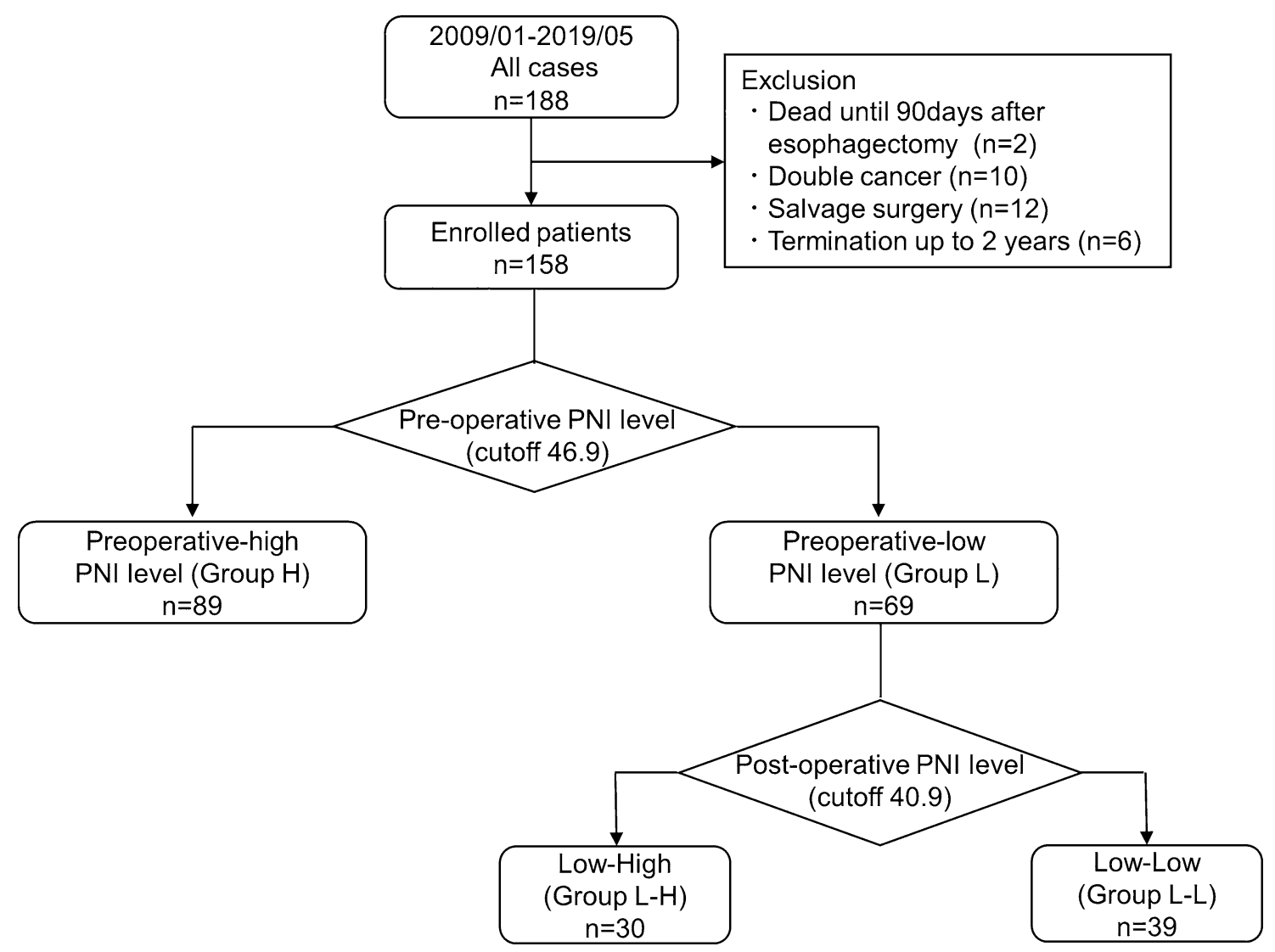

Fig. 1 Flow diagram of the study. Patients enrolled in this study were classified into preoperative-high PNI group (Group H) and preoperative-low PNI group (Group L). Furthermore, based on the postop- erative prognostic nutritional index levels, patients in Group L were divided into preoperative-low and postoperative-high group (Group $\mathrm{L}-\mathrm{H}$ ) and preoperative-low and postoperative-low group (Group L-L) 
surgery. The time-dependent receiver operating characteristic (ROC) analysis of preoperative PNI-based prediction of 5 -year survival revealed that the cutoff value of preoperative PNI was 46.9 (sensitivity $=0.613$, specificity $=0.651$, Youden index $=0.264)$ (Online Resource 1$)$. Moreover, the time-dependent ROC analysis of postoperative PNI-based prediction of 5-year survival revealed that the cutoff value of postoperative PNI was 40.9 (sensitivity $=0.572$, specificity $=0.540$, Youden index $=0.112)($ Online Resource 2$)$. Based on these results, patients were divided into preoperative-high group (Group $\mathrm{H}$ ) and preoperative-low group (Group L). Furthermore, patients in Group L were divided into preoperative-low and postoperative-high group (Group $\mathrm{L}-\mathrm{H}$ ) and preoperative-low and postoperative-low group (Group L-L) (Fig. 1).

\section{Treatment and postoperative complications}

Neoadjuvant chemotherapy was performed as a standard treatment for patients with non-stage I ESCC. The treatment regimen was a combination of cisplatin and 5-fluorouracil or a combination of docetaxel, cisplatin, and 5-fluorouracil. Transthoracic esophagectomy with 2- or 3-field lymph node ( $\mathrm{LN})$ dissection and gastric conduit reconstruction via the posterior mediastinal route was performed as a standard surgical procedure at our institution [16]. In the thoracic approach, video-assisted thoracoscopic surgery in the left decubitus position was generally adopted. Thoracotomy was performed for patients who refused thoracoscopy or were enrolled in another clinical trial. When the stomach could not be used due to a previous history of gastrectomy, reconstruction using the right hemi-colon was performed [16]. Only one patient in Group L-H underwent reconstruction using the right hemi-colon in a two-stage operation because of a history of gastrectomy due to gastric ulcer and scleroderma. Postoperative complications were evaluated for pneumonia, anastomotic leakage (AL), and surgical site infection (SSI) using the Clavien-Dindo classification. Complications of grade 2 or higher were identified as postoperative complications $[17,18]$. The multidisciplinary support team, comprised surgeons, physicians of rehabilitation, nurses, dentists, physiotherapists, speech-language-hearing therapists, and managerial dieticians. This team was set up since April 2017, to prevent complications and ameliorate the postoperative nutritional status of all patients after esophagectomy [17].

\section{Perioperative nutritional support and rehabilitation}

According to our protocol, patients were instructed to abstain from smoking and alcohol consumption for at least 4 weeks before surgery. Physiotherapists commenced respiratory rehabilitation using an incentive spirometer. Following the surgery, early ambulation and restart of respiratory rehabilitation were encouraged. An elemental diet was started at $10 \mathrm{kcal} / \mathrm{h}$ from the day of surgery via a jejunostomy tube for patients with a jejunostomy. The tube-feeding dose was gradually increased to $1200 \mathrm{kcal} /$ day. Enteral nutrition through jejunostomy was continued in the patients, even after the hospital discharge until there were satisfactory results in oral intake [17].

At the time of oral feeding initiation, the rehabilitation physician and the speech-language-hearing therapists performed videofluoroscopic (VF) and videoendoscopic (VE) examinations, especially for the patients receiving multidisciplinary team support. The meals were started with a dysphagia diet. The diet was changed to liquids in accordance with the improvement in the swallowing function. Furthermore, the total calorie intakes were based on decisions made by the multidisciplinary team conference. The calorie intake at the time of discharge was approximately $1500 \mathrm{kcal} / \mathrm{day}$ after introduction of multidisciplinary team support [17]. After the first visit in the outpatient clinic, the dietician evaluated the patients' dietary intake and continued to give nutritional advice regularly.

\section{Follow-up}

Postoperative follow-up was performed using CT every 6 months and EGD every year for 5 years after surgery. Recurrence-free survival (RFS) was calculated from the operation to the day of recurrence of esophageal cancer. Overall survival (OS) was calculated from the operation to the day of death due to esophageal cancer. Patients were followed up until death or until the end of the study (May 31, 2021). Patients who died of an illness unrelated to esophageal cancer, interrupted follow-up, or under following up were recognized as censored, and RFS and OS were calculated based on the days until censoring.

\section{Statistical analysis}

The time-dependent ROC analysis was performed using $\mathrm{R}$ software v 4.0.5 (R Foundation for Statistical Computing, Vienna, Austria [19]). The time-dependent ROC analysis had used to diagnosis 5-year survival in this study. Other statistical analyses were performed using IBM SPSS Statistics version 26 for Windows (Chicago, IL, USA). Medians and ranges were calculated, and differences were identified using Student's $t$ test. The Mann-Whitney $U$ test was used for non-parametric analyses. Differences between each category were identified using the Chi-square test or Fisher's exact test, and the Kruskal-Wallis test was used to identify the differences in continuous variables. Survival curves were produced using the Kaplan-Meier survival method and log-rank test. Hazard ratios (HRs) were calculated, and 
univariate and multivariate analyses were performed using Cox proportional hazards regression models. The threshold for significance was set up at $p<0.05$.

\section{Results}

\section{The impact of preoperative PNI level on survival}

Clinicopathological features between Group H and Group $\mathrm{L}$ are shown in Table 1 . The median follow-up period was 43.4 (4.3-133.5) months. Patients in Group L had a more advanced clinical stage $(p<0.001)$ and, thus, the rate of patients who received neoadjuvant chemotherapy was higher in Group L $(p=0.004)$. The rate of multidisciplinary team support was similar in both groups $(p=0.736)$ (Table 1$)$. Radical esophagectomy with two- or three-field LN dissection was performed in all patients. There was no significant difference in the rate of minimally invasive esophagectomy ( $p=0.338$ ). Moreover, no significant difference was observed in terms of LN dissection, operation time, and jejunostomy (Table 1). The rate of reconstruction organ was similar among both groups. With postoperative complications, the prevalence of postoperative pneumonia was significantly higher in Group L than that in Group H (39.1\% vs. $23.6 \%, p=0.038)$. There was no significant difference in AL and SSI between Groups L and H (AL 20.3\% vs. 21.3\%, $p=1.000$; SSI $29.0 \%$ vs. $30.3 \%, p=1.000$ ). Pathological study showed that the patients in Group L were diagnosed at an advanced stage $(p=0.002)$. The rate of patients who received either adjuvant chemotherapy or radiation was higher in Group L than those in Group H $(p=0.012)$. There was no significant difference in the proportion of local recurrence between the groups. However, the rates of patients who had regional LN and distant organ recurrence were significantly higher in Group L than in Group $\mathrm{H}$ (regional LN recurrence: $31.9 \%$ vs $17.0 \%, p=0.037$; distant organ recurrence: $34.8 \%$ vs $17.0 \%, p=0.015$, respectively) (Table 1 ).

In Kaplan-Meier analysis, the OS and RFS were significantly worse in Group L than in Group $\mathrm{H}(p=0.001$, $p=0.003$ ) (Fig. 2).

\section{The impact of changes in PNI level from preoperative to postoperative phase on survival}

To confirm the correlation between the changes in PNI level from the preoperative to postoperative phase and survival, the survival rates among the three groups were compared. Clinicopathological features between Groups L-H and L-L are shown in Table 2. There was no significant difference in clinical stage between Groups $\mathrm{L}-\mathrm{H}$ and $\mathrm{L}-\mathrm{L}(p=0.132)$. The proportion of multidisciplinary team support in Group
L-L tended to be lower than that in Group L-H (25.6\% vs. $40.0 \%, p=0.298)$. The incidence of postoperative infectious complications (AL, pneumonia, and SSI) was higher in Group L-L than in Group L-H $(p=0.032)$. The prevalence of AL and SSI was significantly higher in Group L-L than those in Group L-H (AL: $30.8 \%$ vs. $6.7 \%, p=0.016$; SSI: $41.0 \%$ vs. $13.3 \%, p=0.016)$. The rate of patients who received enteral nutrition in the early postoperative phase was similar between the groups $(53.3 \%$ in Group L-H vs. $51.3 \%$ in Group $\mathrm{L}-\mathrm{L}, p=1.000)$. There was no difference in the population of patients with death unrelated to esophageal cancer $(p=1.000)$. The proportion of patients who received adjuvant chemotherapy was similar between the two groups $(p=0.336)$. However, adjuvant therapy was suspended in two patients from Group L-L due to adverse events (Table 2).

In the Kaplan-Meier analysis, the RFS in Group L-H tended to be better than those in Group L-L $(p=0.077)$. Furthermore, patients in Group L-H had significantly better OS than those in Group L-L ( $p=0.023)$ (Fig. 3). Patients in Group L-L had significantly worse OS and RFS than those in Group $\mathrm{H}(p<0.001, p<0.001)$. However, there was no significant difference in OS and RFS between Groups L-H and $\mathrm{H}(p=0.224, p=0.161)$ (Fig. 3).

\section{Risk factors for poor prognosis after esophagectomy}

All of the variables shown in Table 3 were potentially significant clinicopathologic variables of prognosis in patients with ESCC. In univariate analysis, neoadjuvant chemotherapy, advanced pathological stage $(\geq \mathrm{II})$, and Group L-L were associated with a worse OS $(p=0.004$, $p<0.001$, and $p<0.001$, respectively) (Table 3). Postoperative complications (AL, pneumonia, and SSI) were not associated with OS ( $p=0.548)$ (Table 3$)$. In multivariate analysis, advanced pathological stage (HR $10.947 ; 95 \%$ CI 2.590-46.268; $p=0.001$ ) and Group L-L (HR 2.171; 95\% CI $1.249-3.775 ; p=0.006)$ were independent predictors of worse OS (Table 3 ).

\section{Effect of multidisciplinary team support}

A stratified analysis before and after introduction of multidisciplinary team support was performed. The proportion of patients in Group L-H increased markedly from $17.1 \%$ before initiation of the multidisciplinary team support to $22.6 \%$ after initiation of the multidisciplinary team support (Online Resource 3). Before introduction of multidisciplinary team support, patients in Group L-H showed significantly better OS and RFS than those in Group L-L (median survival time (MST); OS: 61.1 months vs. 35.4 months; RFS: 61.1 months vs. 24.4 months). Furthermore, there was no difference in the OS and RFS between Groups 
Table 1 Clinicopathological features between Groups $\mathrm{H}$ and L

\begin{tabular}{|c|c|c|c|c|}
\hline & All cases, $n=158$ & Group $\mathrm{H}, n=89$ & Group L, $n=69$ & $p$ value \\
\hline Age (median, years) $\dagger$ & $67(40-82)$ & $67(42-81)$ & $67(40-82)$ & 0.702 \\
\hline Gender (\%) & & & & 0.207 \\
\hline Male & $140(88.6 \%)$ & $76(85.4 \%)$ & $64(92.8 \%)$ & \\
\hline Female & $18(11.4 \%)$ & $13(14.6 \%)$ & $5(7.2 \%)$ & \\
\hline Preoperative body weight (median, $\mathrm{kg}$ ) $\dagger$ & $57.6(36.0-84.4)$ & $57.4(36.0-80.3)$ & $57.7(36.6-84.4)$ & 0.673 \\
\hline Preoperative BMI (median, $\left.\mathrm{kg} / \mathrm{m}^{2}\right) \dagger$ & $21.1(14.2-29.0)$ & $21.3(14.2-29.0)$ & $20.7(14.2-28.9)$ & 0.142 \\
\hline Location of tumor (\%) & & & & 0.943 \\
\hline Ut & $16(10.1 \%)$ & $9(10.1 \%)$ & $7(10.1 \%)$ & \\
\hline Mt & $89(56.3 \%)$ & $49(55.1 \%)$ & $40(58.0 \%)$ & \\
\hline Lt and Ae & $53(33.5 \%)$ & $31(34.8 \%)$ & $22(31.9 \%)$ & \\
\hline Clinical stage, TNM 8th (\%) & & & & $<0.001$ \\
\hline Stage I & $65(41.1 \%)$ & $50(56.2 \%)$ & $15(21.7 \%)$ & \\
\hline Stage II & $49(31.0 \%)$ & $24(27.0 \%)$ & $25(36.2 \%)$ & \\
\hline Stage III & $39(24.7 \%)$ & $15(16.9 \%)$ & $24(34.8 \%)$ & \\
\hline Stage IVA & $5(3.2 \%)$ & $0(0.0 \%)$ & $5(7.2 \%)$ & \\
\hline Preoperative therapy $(\%)$ & & & & 0.004 \\
\hline None & $78(49.4 \%)$ & $53(59.6 \%)$ & $44(63.8 \%)$ & \\
\hline NAC & $80(50.6 \%)$ & $36(40.4 \%)$ & $25(36.2 \%)$ & \\
\hline Multidisciplinary team support (\%) & $53(33.5 \%)$ & $31(34.8 \%)$ & $22(31.9 \%)$ & 0.736 \\
\hline Preoperative serum albumin $\dagger$ & $4.1(2.9-4.9)$ & $4.2(3.8-4.9)$ & $3.7(2.9-4.3)$ & $<0.001$ \\
\hline Preoperative serum total lymphocyte count $\dagger$ & $1425(539-3501)$ & $1575(818-3501)$ & $1271(539-2366)$ & $<0.001$ \\
\hline Preoperative $\mathrm{PNI} \dagger$ & $47.7(32.2-62.7)$ & $50.9(46.9-62.7)$ & $40.2(20.9-51.3)$ & $<0.001$ \\
\hline Surgical approach (\%) & & & & 0.338 \\
\hline Thoracotomy & $76(48.1 \%)$ & $46(51.7 \%)$ & $30(43.5 \%)$ & \\
\hline MIE & $82(51.9 \%)$ & $43(48.3 \%)$ & $39(56.5 \%)$ & \\
\hline $\mathrm{LN}$ dissection $(\%)$ & & & & 0.802 \\
\hline 2-field & $18(11.4 \%)$ & $11(12.4 \%)$ & $7(10.1 \%)$ & \\
\hline 3 -field & $140(88.6 \%)$ & $78(87.6 \%)$ & $62(89.9 \%)$ & \\
\hline Reconstruct organ (\%) & & & & 0.105 \\
\hline Gastric conduit & $148(93.7 \%)$ & $86(96.6 \%)$ & $62(89.9 \%)$ & \\
\hline Colon conduit & $10(6.3 \%)$ & $3(3.4 \%)$ & $7(10.1 \%)$ & \\
\hline Jejunostomy (\%) & $78(49.4 \%)$ & $42(47.2 \%)$ & $36(52.2 \%)$ & 0.631 \\
\hline Operation time (median, min) $\dagger$ & $603(318-1008)$ & $603(347-1008)$ & $618(318-982)$ & 0.611 \\
\hline \multicolumn{5}{|l|}{ Complications, $\mathrm{C}-\mathrm{D}$ grade, $\geq 2(\%)$} \\
\hline All infectious complications & $80(50.6 \%)$ & $42(47.2 \%)$ & $38(55.1 \%)$ & 0.341 \\
\hline $\mathrm{AL}$ & $33(20.9 \%)$ & $19(21.3 \%)$ & $14(20.3 \%)$ & 1.000 \\
\hline Pneumonia & $48(30.4 \%)$ & $21(23.6 \%)$ & $27(39.1 \%)$ & 0.038 \\
\hline SSI & $47(29.7 \%)$ & $27(30.3 \%)$ & $20(29.0 \%)$ & 1.000 \\
\hline Pathological stage, TNM 8th (\%) & & & & 0.002 \\
\hline Stage 0 & $4(2.5 \%)$ & $1(1.1 \%)$ & $3(4.3 \%)$ & \\
\hline Stage IA/IB & $45(28.5 \%)$ & $36(40.4 \%)$ & $9(13.0 \%)$ & \\
\hline Stage IIA/IIB & $34(21.5 \%)$ & $18(20.2 \%)$ & $16(23.2 \%)$ & \\
\hline Stage IIIA/IIIB & $50(31.7 \%)$ & $23(25.8 \%)$ & $27(39.1 \%)$ & \\
\hline Stage IVA/IVB & $25(15.8 \%)$ & $11(12.4 \%)$ & $14(20.3 \%)$ & \\
\hline Adjuvant therapy (\%) & & & & 0.012 \\
\hline None & $92(58.2 \%)$ & $59(66.3 \%)$ & $32(46.4 \%)$ & \\
\hline Chemotherapy & $65(63.3 \%)$ & $29(32.6 \%)$ & $37(53.6 \%)$ & \\
\hline Radiation & $1(0.6 \%)$ & $1(1.1 \%)$ & $0(0.0 \%)$ & \\
\hline POM1 serum albumin $\dagger$ & $3.5(1.6-4.6)$ & $3.6(2.0-4.6)$ & $3.5(1.6-4.4)$ & 0.014 \\
\hline POM1 serum total lymphocyte count $\dagger$ & $1183(300-3107)$ & $1261(402-3107)$ & $1023(300-2357)$ & 0.003 \\
\hline
\end{tabular}


Table 1 (continued)

\begin{tabular}{|c|c|c|c|c|}
\hline & All cases, $n=158$ & Group H, $n=89$ & Group L, $n=69$ & $p$ value \\
\hline POM1 PNI $\dagger$ & $41.1(20.9-56.5)$ & $42.9(23.4-56.5)$ & $40.2(20.9-51.3)$ & 0.003 \\
\hline \multicolumn{5}{|l|}{ Recurrence site* (\%) } \\
\hline Local & $12(7.6 \%)$ & $5(5.7 \%)$ & $7(10.1 \%)$ & 0.369 \\
\hline Regional LN & $37(23.6 \%)$ & $15(17.0 \%)$ & $22(31.9 \%)$ & 0.037 \\
\hline Distant organ & $39(24.8 \%)$ & $15(17.0 \%)$ & $24(34.8 \%)$ & 0.015 \\
\hline Death unrelated to esophageal cancer (\%) & $8(5.1 \%)$ & $6(6.7 \%)$ & $2(2.9 \%)$ & 0.467 \\
\hline
\end{tabular}

$B M I$ body mass index, $U t$ upper thoracic esophagus, $M t$ middle thoracic esophagus, $L t$ lower thoracic esophagus, Ae abdominal esophagus, $N A C$ neoadjuvant chemotherapy, $P N I$ prognostic nutritional index, $M I E$ minimal invasive esophagectomy, $L N$ lymph node, $C-D C l a v i e n-D i n d o, A L$ anastomotic leakage, SSI surgical site infection, POM postoperative month

${ }^{\dagger}$ Values are presented as median (range)

* Some patients were existed multiple sites of recurrence

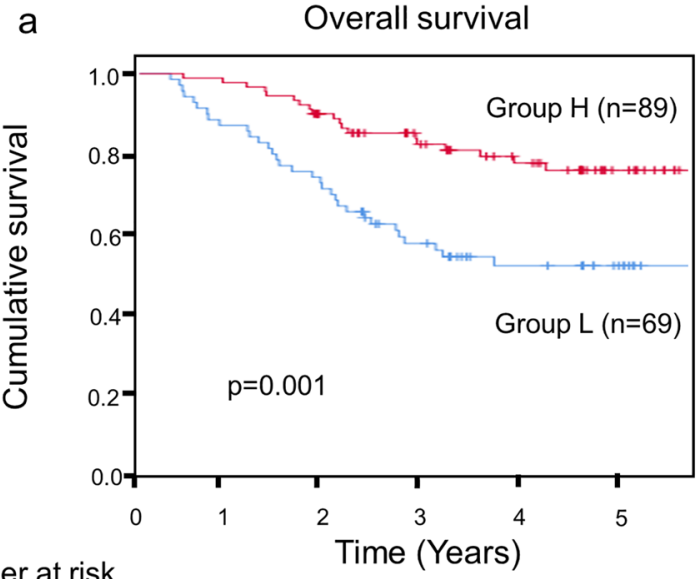

Number at risk

$\begin{array}{lllllll}\text { Group H } & 89 & 87 & 80 & 65 & 49 & 41 \\ \text { Group L } & 69 & 60 & 51 & 36 & 24 & 22\end{array}$

b

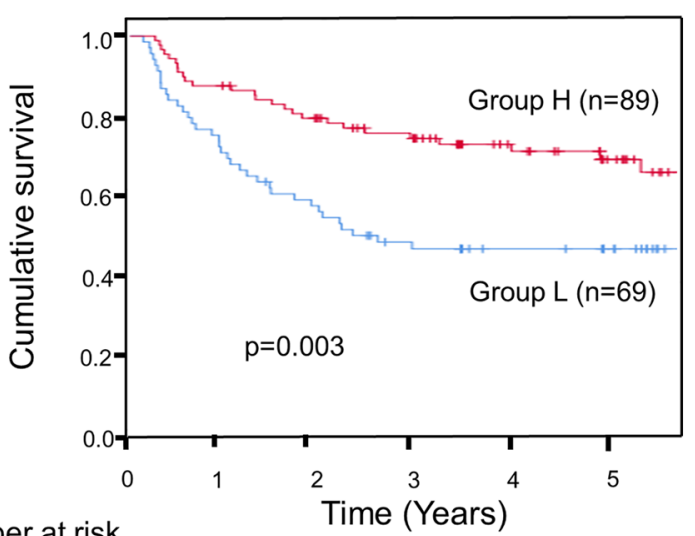

Number at risk

$\begin{array}{lllllll}\text { Group H } & 89 & 77 & 68 & 59 & 43 & 37 \\ \text { Group L } & 69 & 49 & 39 & 29 & 22 & 21\end{array}$

Fig. 2 Kaplan-Meier analysis with preoperative prognostic nutritional index levels. a Comparison of overall survival. b Comparison of recurrence free survival

$\mathrm{L}-\mathrm{H}$ and $\mathrm{H}$. Although the number of patients who received multidisciplinary team support was small, after it was initiated, patients in Group L-H also tended to have a good OS than those in Group L-L (MST of OS: 36.2 months vs. 23.7 months). Moreover, the survival curves were also similar between Groups L-H and H (Online Resource 3).

\section{Survival impact of postoperative nutritional support for patients with infectious complications}

To investigate the survival impact postoperative nutritional support, the long-term outcomes of patients with postoperative infectious complications (AL, pneumonia, and SSI) were assessed using a stratified analysis which patients were divided into groups based on whether they developed postoperative infectious complications (Online Resource 4). In the stratified analysis, either with or without postoperative infectious complications, patients in Group L-H were tended to have better OS and RFS than those in Group L-L (Online Resource 4).

\section{Discussion}

This study demonstrated that preoperative-low PNI patients who did not sufficiently recover in terms of postoperative PNI level had a significantly worse OS. In contrast, preoperative-low PNI patients who sufficiently recovered in terms of postoperative PNI levels had similar OS to those with preoperative-high PNI levels. These results suggested that the 
Table 2 Clinicopathological features between Groups L-H and $\mathrm{L}-\mathrm{L}$

\begin{tabular}{|c|c|c|c|}
\hline & Group L-H, $n=30$ & Group L-L, $n=39$ & $p$ value \\
\hline Age (median, years) $\dagger$ & $66.5(48-76)$ & $67(40-82)$ & 0.216 \\
\hline Gender (\%) & & & 0.159 \\
\hline Male & $26(86.7 \%)$ & $38(97.4 \%)$ & \\
\hline Female & $4(13.3 \%)$ & $1(2.6 \%)$ & \\
\hline Location of tumor (\%) & & & 0.593 \\
\hline Ut & $2(6.7 \%)$ & $5(12.8 \%)$ & \\
\hline Mt & $17(56.7 \%)$ & $23(59.0 \%)$ & \\
\hline $\mathrm{Lt}$ and $\mathrm{Ae}$ & $11(36.7 \%)$ & $11(28.2 \%)$ & \\
\hline Clinical stage, TNM 8th (\%) & & & 0.132 \\
\hline Stage I & $10(33.3 \%)$ & $5(12.8 \%)$ & \\
\hline Stage II & $8(26.7 \%)$ & $17(43.6 \%)$ & \\
\hline Stage III & $11(36.7 \%)$ & $13(33.3 \%)$ & \\
\hline Stage IVA & $1(3.3 \%)$ & $4(10.3 \%)$ & \\
\hline Preoperative therapy (\%) & & & 1.000 \\
\hline None & $11(36.7 \%)$ & $14(35.9 \%)$ & \\
\hline NAC & $19(63.3 \%)$ & $25(64.1 \%)$ & \\
\hline Multidisciplinary team support (\%) & $12(40.0 \%)$ & $10(25.6 \%)$ & 0.298 \\
\hline Surgical approach (\%) & & & 1.000 \\
\hline Thoracotomy & $13(43.3 \%)$ & $17(43.6 \%)$ & \\
\hline MIE & $17(56.7 \%)$ & $22(56.4 \%)$ & \\
\hline LN dissection (\%) & & & 0.128 \\
\hline 2-field & $1(3.3 \%)$ & $6(15.4 \%)$ & \\
\hline 3 -field & $29(96.7 \%)$ & $33(84.6 \%)$ & \\
\hline Reconstruct organ (\%) & & & 0.690 \\
\hline Gastric conduit & $26(86.7 \%)$ & $36(92.3 \%)$ & \\
\hline Colon conduit & $4(13.3 \%)$ & $3(7.7 \%)$ & \\
\hline Jejunostomy (\%) & $16(53.3 \%)$ & $20(51.3 \%)$ & 1.000 \\
\hline Operation time (median, min) $\dagger$ & $559.5(318-982)$ & $645(441-927)$ & 0.055 \\
\hline \multicolumn{4}{|l|}{ Complications, C-D grade, $\geq 2(\%)$} \\
\hline All infectious complications & $12(40.0 \%)$ & $26(66.7 \%)$ & 0.032 \\
\hline AL & $2(6.7 \%)$ & $12(30.8 \%)$ & 0.016 \\
\hline Pneumonia & $10(33.3 \%)$ & $17(43.6 \%)$ & 0.460 \\
\hline SSI & $4(13.3 \%)$ & $16(41.0 \%)$ & 0.016 \\
\hline Pathological stage, TNM 8th (\%) & & & 0.320 \\
\hline Stage 0 & $3(10.0 \%)$ & $0(0.0 \%)$ & \\
\hline Stage IA/IB & $5(16.7 \%)$ & $4(10.3 \%)$ & \\
\hline Stage IIA/IIB & $6(20.0 \%)$ & $10(25.6 \%)$ & \\
\hline Stage IIIA/IIIB & $11(36.7 \%)$ & $16(37.9 \%)$ & \\
\hline Stage IVA/IVB & $5(16.7 \%)$ & $9(23.1 \%)$ & \\
\hline Adjuvant therapy (\%) & & & 0.336 \\
\hline None & $13(43.3 \%)$ & $22(56.4 \%)$ & \\
\hline Chemotherapy & $17(56.7 \%)$ & $17(43.6 \%)$ & \\
\hline POM1 serum albumin $\dagger$ & $3.8(3.3-4.4)$ & $3.0(1.6-3.7)$ & $<0.001$ \\
\hline POM1 serum total lymphocyte count $\dagger$ & $1218.5(590-2357)$ & $972(300-2244)$ & 0.011 \\
\hline POM1 PNI $\dagger$ & $44.5(40.9-51.3)$ & $35.1(20.9-40.8)$ & $<0.001$ \\
\hline \multicolumn{4}{|l|}{ Recurrence site* (\%) } \\
\hline Local & $2(6.7 \%)$ & $5(12.8 \%)$ & 0.690 \\
\hline Regional LN & $6(20.0 \%)$ & $16(41.0 \%)$ & 0.074 \\
\hline Distant organ & $8(26.7 \%)$ & $16(41.0 \%)$ & 0.308 \\
\hline Death unrelated to esophageal cancer (\%) & $1(3.3 \%)$ & $1(2.6 \%)$ & 1.000 \\
\hline
\end{tabular}

$U t$ Upper thoracic esophagus, $M t$ middle thoracic esophagus, $L t$ lower thoracic esophagus; Ae Abdominal esophagus, NAC neoadjuvant chemotherapy, MIE minimal invasive esophagectomy, $L N$ lymph node, $C-D$ Clavien-Dindo, $A L$ anastomotic leakage, SSI surgical site infection, $P O M$ postoperative month, $P N I$ prognostic nutritional index

†Values are presented as median (range)

*Some patients were existed multiple sites of recurrence 
a

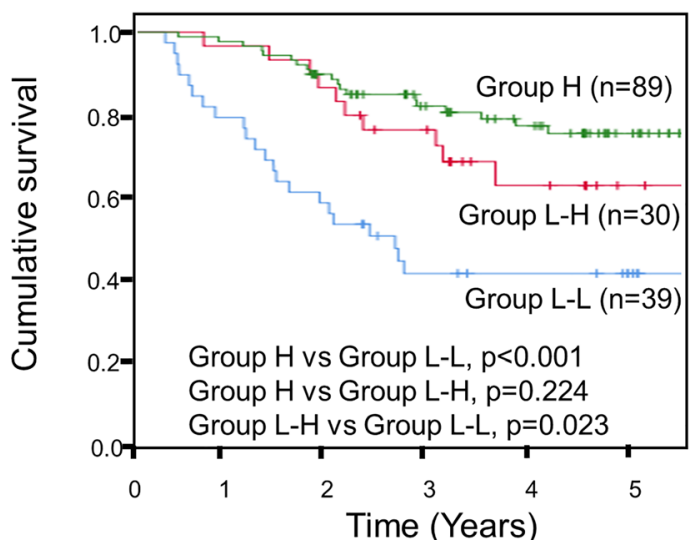

Number at risk

$\begin{array}{lllllll}\text { Group H } & 89 & 87 & 80 & 65 & 49 & 41 \\ \text { Group L-H } & 30 & 29 & 26 & 21 & 12 & 10 \\ \text { Group L-L } & 39 & 31 & 24 & 15 & 12 & 12\end{array}$

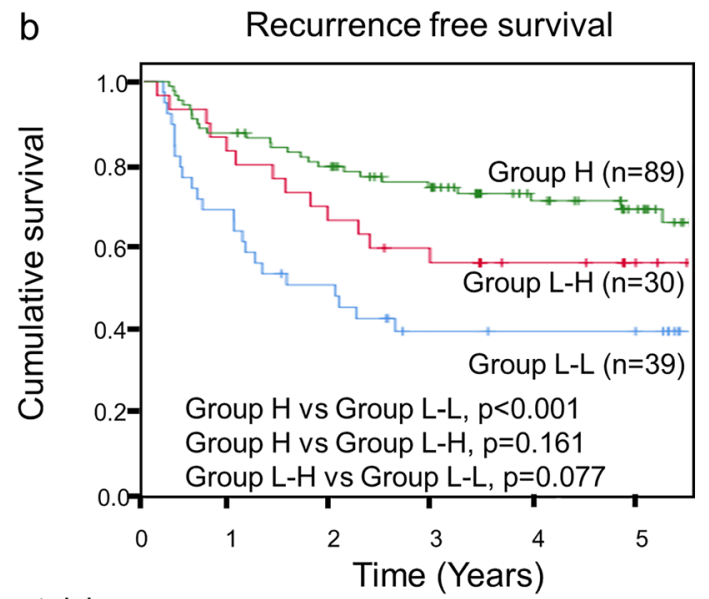

Number at risk

$\begin{array}{lllllll}\text { Group H } & 89 & 77 & 68 & 59 & 43 & 37 \\ \text { Group L-H } & 30 & 24 & 20 & 17 & 11 & 10 \\ \text { Group L-L } & 39 & 25 & 19 & 12 & 11 & 11\end{array}$

Fig. 3 Kaplan-Meier analysis of the changes in prognostic nutritional index levels from preoperative to postoperative phase. a Comparison of overall survival between the three groups. b Comparison of recurrence free survival between the three groups

Table 3 Independent factors of clinicopathological, surgical, and pathological features on shorter overall survival

\begin{tabular}{|c|c|c|c|c|c|c|}
\hline & \multicolumn{3}{|c|}{ Univariate analysis } & \multicolumn{3}{|c|}{ Multivariate analysis } \\
\hline & HR & $p$ & $95 \% \mathrm{CI}$ & HR & $p$ & $95 \% \mathrm{CI}$ \\
\hline Age $(>66$ vs $\leq 66)$ & 0.962 & 0.889 & $0.561-1.650$ & & & \\
\hline Transthoracic approach (thoracotomy vs MIE) & 1.041 & 0.883 & $0.607-1.785$ & & & \\
\hline Reconstruction (colon conduit vs gastric conduit) & 2.093 & 0.090 & $0.891-4.915$ & & & \\
\hline All infectious complications (+vs -) & 1.181 & 0.548 & $0.686-2.035$ & & & \\
\hline $\mathrm{AL}(+\mathrm{vs}-)$ & 1.114 & 0.736 & $0.595-2.084$ & & & \\
\hline Pneumonia (+vs -) & 1.518 & 0.138 & $0.874-2.635$ & & & \\
\hline SSI (+ vs -) & 1.224 & 0.481 & $0.698-2.147$ & & & \\
\hline Adjuvant therapy (+ vs -) & 1.383 & 0.238 & $0.807-2.371$ & & & \\
\hline Neoadjuvant chemotherapy (+vs -) & 2.301 & 0.004 & $1.302-4.067$ & 1.353 & 0.306 & $0.758-2.416$ \\
\hline Pathological stage ( $\geq$ II vs < II) & 14.803 & $<0.001$ & $3.601-60.857$ & 10.947 & 0.001 & $2.590-46.268$ \\
\hline Change of PNI level (Group L-L vs other groups) & 3.022 & $<0.001$ & $1.746-5.228$ & 2.171 & 0.006 & $1.249-3.775$ \\
\hline
\end{tabular}

$H R$ hazard ratio, $C I$ confidence interval, $M I E$ minimally invasive esophagectomy, $A L$ anastomotic leakage, $S S I$ surgical site infection, $P N I$ prognostic nutritional index

changes in PNI levels from the pre- to postoperative phase may predict the prognosis in patients with ESCC, and successful recovery of PNI level after transthoracic esophagectomy can improve the OS in preoperative-low PNI patients to match that of preoperative-high PNI patients.

PNI was initially proposed by Buzby [20] and modified by Onodera [12]. PNI, which includes albumin level and lymphocyte counts in its calculation, is a combined simple index of nutrition and immune status. Low albumin levels and lymphocyte counts reflect systemic inflammation in cancer patients. For several types of cancer, including pancreatic cancer, hepatocellular carcinoma, gastric cancer, and colon cancer, PNI was shown to be a significant prognostic factor. Previous studies have reported that preoperative PNI level was associated with prognosis for ESCC [14, 21, 22]. However, to the best of our knowledge, this is the first report on the survival impact of the changes in PNI level during the perioperative period after esophagectomy. In this study, postoperative PNI was set from blood test of 1 month later. At acute phase after surgery, intensive invasion and systemic 
inflammation persisted, and these factors might inhibit the effect of nutritional support. In contrast, some patients in this study received adjuvant therapy within 3 months after surgery, which might affect the PNI level.

The controlling nutritional status (CONUT) score is another representative nutritional index which reported as an independent predictor of prognosis of esophageal cancer [23]. Evaluation of the association between the preoperative PNI levels and the preoperative CONUT score was performed in this dataset. In this study, the preoperative PNI levels was significantly lower in the high CONUT score group (CONUT score 2 or higher) than those in the low CONUT score group (CONUT score 0-1) (data not shown). These results suggested that there was a correlation between both nutritional indexes. On the other hand, Lin, et al. compared the association of the PNI level and CONUT score with prognosis of gastric cancer patients, and reported that the CONUT score was important in assessing the risk of severe postoperative morbidity, and the PNI level associated more precisely with long-term prognosis [24]. That is why the PNI level was used as an indicator in this study.

In this study, patients in Group L-L tended to have more frequent postoperative complications than those in Group L-H. Postoperative complications reportedly induced the suppression of albumin production and elevation of white blood cells, particularly neutrophils, resulting in insufficient PNI recovery [25]. Interestingly, the stratified analysis for patients with postoperative infectious complications demonstrated that patients in Group L-L were tended to have poor OS and RFS than those in Group L-H. Furthermore, there was no correlation between postoperative complications and OS from the univariable Cox regression analysis. These results suggested that preventing a decline of PNI level could improve the prognosis, even in patients with postoperative complications.

The proportion of patients in Group L-H increased after introduction of multidisciplinary team support. In the stratified analysis, patients in Group L-L were tended to have poor OS than those in Group $\mathrm{L}-\mathrm{H}$ both before and after introduction of multidisciplinary team support. These results indicated that intensive postoperative care with a multidisciplinary team support could contribute not only to the prevention of postoperative complications and maintenance of PNI levels, but also to the prolongation of prognosis, especially in preoperative-low PNI level patients. Moreover, two patients in Group L-L stopped adjuvant chemotherapy. These cases suggested that sufficient PNI recovery after esophagectomy improved tolerance to adjuvant chemotherapy. In multidisciplinary team support, team conference in which the members could share the patients' nutritional status, swallowing function from VF and VE, and calorie intake could have contributed to the enhanced nutritional support [17]. Moreover, regular nutritional counseling in outpatient clinic also could support patients' nutritional status [17].

Patients in Group L-L had significantly worse RFS than the others did, and patients in Group L-H had similar RFS to those in Group H. However, when limited to the first year after esophagectomy, the decline in RFS rate was similar between Groups L-H and L-L. After a year, the RFS rate continued to decline in Group L-L, but that in Group $\mathrm{L}-\mathrm{H}$ was relatively stable. These results suggested that postoperative PNI reflected tumor immunity. Okadome et al. reported that the systemic nutritional and immunological status in patients may be associated with local tumor immunity by evaluating the relationship between PNI and tumor-infiltrating lymphocyte status [13].

The study had some limitations. First, this was a retrospective study conducted in a single institution. However, this study reviewed consecutive patients at our institution, which reduced selection bias. Another limitation is that the study period was relatively long. Consequently, the treatment of patients with ESCC, including the maturation of surgical techniques, regimen of neoadjuvant chemotherapy, and perioperative management, could have changed over this period. There were some biases in the operation and perioperative interventions. Hence, a multi-institutional prospective study must be conducted to validate the current results.

In conclusion, the recovery of PNI levels after esophagectomy could improve the prognosis in patients with preoperative malnutrition. Further studies are warranted to validate this classification and investigate the survival benefit of PNI changes during esophagectomy.

Supplementary Information The online version contains supplementary material available at https://doi.org/10.1007/s10388-021-00883-5.

Acknowledgements The authors gratefully acknowledge the work of the members of the Hamamatsu Perioperative Care Team with collection of clinical data and the support of statistical analysis by Akifumi Nozu, Clinical Research Center, Shizuoka Cancer Center.

\section{Declarations}

Ethical statement All procedures were conducted in accordance with institutional and national standards on human experimentation, as confirmed by the ethics committee of Hamamatsu University School of Medicine (Approval No. 19-098), and with the Declaration of Helsinki of 1964 and its later versions.

Conflict of interest The authors declare no conflicts of interest for this article.

Informed consent Informed consent was obtained from all the subjects.

Open Access This article is licensed under a Creative Commons Attribution 4.0 International License, which permits use, sharing, adaptation, distribution and reproduction in any medium or format, as long 
as you give appropriate credit to the original author(s) and the source, provide a link to the Creative Commons licence, and indicate if changes were made. The images or other third party material in this article are included in the article's Creative Commons licence, unless indicated otherwise in a credit line to the material. If material is not included in the article's Creative Commons licence and your intended use is not permitted by statutory regulation or exceeds the permitted use, you will need to obtain permission directly from the copyright holder. To view a copy of this licence, visit http://creativecommons.org/licenses/by/4.0/.

\section{References}

1. Torre LA, Bray F, Siegel RL, et al. Global cancer statistics, 2012. CA Cancer J Clin. 2015;65:87-108.

2. Ando N, Kato H, Igaki H, et al. A randomized trial comparing postoperative adjuvant chemotherapy with cisplatin and 5-fluorouracil versus preoperative chemotherapy for localized advanced squamous cell carcinoma of the thoracic esophagus (JCOG9907). Ann Surg Oncol. 2012;19:68-74.

3. Kitagawa Y, Uno T, Oyama T, et al. Esophageal cancer practice guidelines 2017 edited by the Japan Esophageal Society: part 1 . Esophagus. 2019;16:1-24.

4. Kitagawa Y, Uno T, Oyama T, et al. Esophageal cancer practice guidelines 2017 edited by the Japan Esophageal society: part 2. Esophagus. 2019;16:25-43

5. Takeuchi H, Miyata H, Ozawa S, et al. Comparison of short-term outcomes between open and minimally invasive esophagectomy for esophageal cancer using a Nationwide Database in Japan. Ann Surg Oncol. 2017;24:1821-7.

6. Kubo N, Ohira M, Tamura T, et al. Prognostic significance of baseline nutritional index for patients with esophageal squamous cell carcinoma after radical esophagectomy. Esophagus. 2016;14:84-90.

7. Booka E, Takeuchi H, Suda K, et al. Meta-analysis of the impact of postoperative complications on survival after oesophagectomy for cancer. BJS Open. 2018;2:276-84.

8. Takesue T, Takeuchi H, Ogura M, et al. A prospective randomized trial of enteral nutrition after thoracoscopic esophagectomy for esophageal cancer. Ann Surg Oncol. 2015;22(Suppl 3):S802-809.

9. Takeuchi H, Ikeuchi S, Kawaguchi Y, et al. Clinical significance of perioperative immunonutrition for patients with esophageal cancer. World J Surg. 2007;31:2160-7.

10. Matsuda S, Takeuchi H, Kawakubo H, et al. Cumulative prognostic scores based on plasma fibrinogen and serum albumin levels in esophageal cancer patients treated with transthoracic esophagectomy: comparison with the Glasgow Prognostic Score. Ann Surg Oncol. 2014;22:302-10.

11. Sharaiha RZ, Halazun KJ, Mirza F, et al. Elevated preoperative neutrophil:lymphocyte ratio as a predictor of postoperative disease recurrence in esophageal cancer. Ann Surg Oncol. 2011;18:3362-9.
12. Onodera T, Goseki N, Kosaki G. Prognostic nutritional index in gastrointestinal surgery of malnourished cancer patients. Nihon Geka Gakkai Zasshi. 1984;85:1001-5.

13. Okadome K, Baba Y, Yagi T, et al. Prognostic nutritional index, tumor-infiltrating lymphocytes, and prognosis in patients with esophageal cancer. Ann Surg. 2020;271:693-700.

14. Liao G, Zhao Z, Yang H, et al. Can prognostic nutritional index be a prediction factor in esophageal cancer? A meta-analysis. Nutr Cancer. 2020;72:187-93.

15. Brierley JDGM, Wittekind C. TNM classification of malignant tumors, 8th edn. 2017.

16. Kikuchi H, Hiramatsu Y, Matsumoto T, et al. The hybrid position is superior to the prone position for thoracoscopic esophagectomy with upper mediastinal lymphadenectomy. Ann Laparosc Endosc Surg. 2020;5:13-13.

17. Kawata S, Hiramatsu Y, Shirai Y, et al. Multidisciplinary team management for prevention of pneumonia and long-term weight loss after esophagectomy: a single-center retrospective study. Esophagus. 2020;17:270-8.

18. Dindo D, Demartines N, Clavien PA. Classification of surgical complications: a new proposal with evaluation in a cohort of 6336 patients and results of a survey. Ann Surg. 2004;240:205-13.

19. R Core Team. R: a language and environment for statistical computing. Vienna, Austria: R Foundation for Statistical Computing; 2020. https://www.R-project.org/. Accessed 10 Jan 2020.

20. Buzby GP, Mullen JL, Matthews DC, et al. Prognostic nutritional index in gastrointestinal surgery. Am J Surg. 1980;139:160-7.

21. Li P, Wang X, Lai Y, et al. The prognostic value of pre-treatment prognostic nutritional index in esophageal squamous cell carcinoma: a meta-analysis. Medicine (Baltimore). 2019;98:e15280.

22. Nozoe T, Kimura Y, Ishida M, et al. Correlation of pre-operative nutritional condition with post-operative complications in surgical treatment for oesophageal carcinoma. Eur J Surg Oncol. 2002;28:396-400.

23. Hikage M, Taniyama Y, Sakurai T, et al. The influence of the perioperative nutritional status on the survival outcomes for esophageal cancer patients with neoadjuvant chemotherapy. Ann Surg Oncol. 2019;26:4744-53.

24. Lin JX, Lin LZ, Tang YH, et al. Which nutritional scoring system is more suitable for evaluating the short- or long-term prognosis of patients with gastric cancer who underwent radical gastrectomy? J Gastrointest Surg. 2020;24:1969-77.

25. Matsuda S, Takeuchi H, Kawakubo H, et al. Prognostic impact of change in the Fibrinogen and Albumin Score during preoperative treatment in esophageal cancer patients. World J Surg. 2017;41:2788-95.

Publisher's Note Springer Nature remains neutral with regard to jurisdictional claims in published maps and institutional affiliations. 